\title{
Non-invasive monitoring of anticancer effects of cisplatin on lung cancer in an orthotopic SCID mouse model using $\left[{ }^{18}\right.$ F] FDG PET-CT
}

\author{
MOHAMED MOKHTAR ${ }^{1,2}$, KAZUYA KONDO $^{1}$, HIROMITSU TAKIZAWA $^{3}$, TAMAKI OHTANI $^{4}$, \\ HIDEKI OTSUKA ${ }^{4}$, HITOSHI KUBO ${ }^{4}$, KOICHIRO KAJIURA ${ }^{3}$, YASUSHI NAKAGAWA ${ }^{3}$, \\ YUKIKIYO KAWAKAMI ${ }^{3}$, MITSUTERU YOSHIDA ${ }^{3}$, HARUHIKO FUJINO ${ }^{3}$, \\ SHOJI SAKIYAMA ${ }^{3}$ and AKIRA TANGOKU ${ }^{3}$
}

\begin{abstract}
${ }^{1}$ Department of Oncological Medical Services, Institute of Health Biosciences, The University of Tokushima Graduate School, Tokushima, Japan; ${ }^{2}$ Department of Oncological Surgery, Minia Oncology Institute, Minia, Egypt; ${ }^{3}$ Department of Thoracic, Endocrine and Oncological Surgery, Institute of Health Biosciences, The University of Tokushima Graduate School, Tokushima; ${ }^{4}$ Department of Medical Imaging, The University of Tokushima Graduate School, Tokushima, Japan
\end{abstract}

Received January 8, 2014; Accepted February 10, 2014

DOI: $10.3892 / o r .2014 .3056$

\begin{abstract}
Positron emission tomography-computed tomography (PET-CT) with $\left[{ }^{18} \mathrm{~F}\right]$ fluorodeoxyglucose (FDG) has recently been applied for evaluating tumor response to anticancer therapy. The aim of the present study was to evaluate the utility of FDG PET-CT in monitoring non-invasively and repeatedly the inhibitory effect of cisplatin (CDDP) on an orthotopic lung cancer model. Validation of in vivo FDG uptake in human lung cancer Ma44-3 cell line in an orthotopic SCID mouse model was carried out. Next, we assessed the use of FDG PET-CT to monitor the response of orthotopic lung cancer to the anticancer effect of CDDP. SCID mice were divided into the CDDP group $(7 \mathrm{mg} / \mathrm{kg}$ single dose intraperitoneally) and the control group. Tumor volume and maximal standardized uptake value (SUV max) were calculated for all mice. All mice were sacrificed for histopathologic analysis. Validation of FDG PET-CT showed that tumor volume and SUV max were significantly correlated with postmortem tumor length measured in specimens $(\mathrm{P}=0.023)$ and $(\mathrm{P}=0.012)$, respectively, and there was a significant correlation between SUV max and tumor volume ( $\mathrm{P}=0.048)$. Response monitoring
\end{abstract}

Correspondence to: Professor Kazuya Kondo, Department of Oncological Medical Services, Institute of Health Biosciences, The University of Tokushima Graduate School, 18-15 Kuramoto-cho 3, Tokushima 770-8509, Japan

E-mail: kondo@clin.med.tokushima-u.ac.jp

Abbreviations: PET-CT, positron emission tomography-computed tomography; FDG, fluorodeoxyglucose; CDDP, cisplatin; NSCLC, non-small cell lung cancer; MRI, magnetic resonance imaging; SUV max, maximal standardized uptake value

Key words: positron emission tomography, $\left[{ }^{18} \mathrm{~F}\right]$ fluorodeoxyglucose, small animal imaging, orthotopic model, lung cancer, cisplatin showed that significant growth inhibition by CDDP in the form of SUV max of the CDDP group was significantly lower than that of the control group on day $8(\mathrm{P}=0.02)$ and on day 13 $(\mathrm{P}=0.003)$. Tumor volume of the CDDP group was significantly lower than that of the control group on day $13(\mathrm{P}=0.03)$. The present study supports using FDG PET-CT in monitoring tumor progression and therapeutic response of lung cancer in an orthotopic model non-invasively and repeatedly.

\section{Introduction}

Lung cancer is the most common cause of cancer-related mortality worldwide. The primary reason for the difficulty in treating lung cancer is that it is mostly identified at a very late stage. Lymphogenous or hematogenous metastasis occurs in early stage (1). The majority of patients with advanced nonsmall cell lung cancer (NSCLC) are treated with combination therapy including a platinum-based compound (2). The major barriers limiting the use and efficacy of cisplatin (CDDP) are toxicity and resistance (3). Therefore, finding new therapeutic agents is of great clinical interest in lung cancer research. Preclinical tumor models are a fundamental component of study and design of new regimens for cancer treatment (4). Considerable efforts have been made to develop more clinically relevant models by using orthotopic implantation models. An organ-specific site presumably provides tumor cells with the most appropriate environment for local growth and metastasis. Orthotopic models are likely to provide more relevant pharmacokinetic and pharmacodynamics information than subcutaneous models (5). We established an orthotopic implanted SCID mouse model of lung cancer without thoracotomy (1,6-11). Our model was simple, easy and reproducible. Many models can be produced at once. The main downside of the orthotopic model is that tumor size or volume changes are more difficult to continuously monitor reproducibility, except at necropsy $(5,12)$. Thus, small animal imaging, which is a non-invasive and repeatable method for monitoring the course 
of disease under therapy, has become increasingly important $(13,14)$. One disadvantage of using anatomical imaging techniques such as computed tomography (CT) and magnetic resonance imaging (MRI) in monitoring the tumor size as a response to treatment is the amount of time it requires before a volume response becomes evident. Therefore, new biological measurements suggest using functional fluorodeoxyglucose (FDG) PET for measurement of treatment effects (15). FDG is internalized into cells, where it is subsequently phosphorylated by the enzyme hexokinase. Thus, the accumulation of FDG-6-phosphate observed in PET scans is correlated with cellular glucose metabolism (16). Neoplastic cells usually exhibit increased anaerobic metabolism, which is associated with trapping FDG within the cells (17). Furthermore, the therapeutic response of tumor cells is generally correlated with their metabolic changes and these appear earlier than conventional morphological changes. Thus, functional PET images can document therapeutic success earlier than morphological techniques such as CT and MRI $(18,19)$. In the present study, we evaluated the utility of FDG positron emission tomography-computed tomography (PET-CT) to non-invasively and repeatedly monitor the inhibitory effect of CDDP on lung cancer in an orthotopic SCID mouse model, in order to establish a standard model for examining novel regimens in lung cancer treatment.

\section{Materials and methods}

Animal model. Male SCID mice (CB-17/Icr-scidJc1; Clea Japan, Inc., Tokyo, Japan) 6-8 weeks of age were used in the present study, and were maintained in the Laboratory for Animal Experiments. The protocols of all animal experiments were approved by the Institutional Animal Care and Use Committee of the University of Tokushima, School of Medicine, and were carried out according to their guidelines.

Cells line. Human NSCLC cell lines Ma44 were kindly provided by Dr N. Masuda and Dr Y. Takada (Osaka Prefecture Habikino Hospital, Osaka, Japan). An Ma44-3 cell line was cloned in our laboratory using the limiting dilution method. This cell line was cultured in RPMI-1640 (Sigma Chemical Co., St. Louis, MO, USA) with $10 \%$ heat inactivated fetal bovine serum (BioWhittaker, Walkersville, MD, USA) and maintained at $37^{\circ} \mathrm{C}$ in a humidified incubator with $5 \% \mathrm{CO}_{2}$ in air.

Orthotopic intrapulmonary implantation. As in our previous studies $(1,7,8)$, the mice were fully anesthetized by ether inhalation, and placed in the right lateral decubitus with the four limbs restrained. A $1 \mathrm{~cm}$ transverse incision was made on the left lateral skin just below the inferior border of the scapula of the SCID mouse. Muscles were separated from the ribs by sharp dissection, and intercostal muscles were exposed. The left lung was visible through the intercostal muscles. A 30 -gauge needle was inserted $\sim 5 \mathrm{~mm}$ into the lung through the intercostal muscle, and an inoculum of $2 \times 10^{6}$ tumor cells $/ \mathrm{ml}$ with $400 \mathrm{mg} / \mathrm{ml}$ Matrigel (Collaborative Biomedical Products, Bedford, Canada) was then dispersed into the left lung in a final volume of $10 \mu \mathrm{l}\left(2 \times 10^{4}\right.$ cells) medium. The procedure required $\sim 1$ min for completion and was easily performed. The skin incision was closed with 3-0 silk. In validation of in vivo
FDG uptake, orthotopic implantation was carried out in the left lung of the mice. In monitoring of in vivo response of CDDP and control group, the implantation was performed in the right lung to clearly delineate tumor uptake from heart uptake.

Micro PET and micro CT imaging. The mice were injected intravenously with $10.0 \pm 0.3$ (means \pm SD) MBq FDG. Mice were fasted overnight before each FDG PET scan (20). One hour after tracer injection, mice were anesthetized with $3 \%$ sevofluran (Abbott Scandinavia AB, Solna, Sweden) mixed with $35 \% \mathrm{O}_{2}$ in $\mathrm{N}_{2}$ and fixed on a bed in the presence of three fiducial markers allowing fusion of PET and CT images. A PET scan was acquired using a micro PET Focus 120 followed by a micro CT scan acquired with a micro CATWII system (both from Siemens Medical Solutions, Malvern, PA, USA), as previously described (21). PET data were arranged into sinograms and subsequently reconstructed with the maximum a posteriori (MAP) reconstruction algorithm. The pixel size was $0.866 \times 0.866 \times 0.796 \mathrm{~mm}$ and in the center field of view the resolution was $1.2 \mathrm{~mm}$ full-width-at-half-maximum. PET and micro CT images were fused in the Inveon software (Siemens Medical Solutions). Before fusion, regions of interest (ROIs) were drawn on the CT images manually by qualitative assessment covering the whole tumors and subsequently tumor volume and tracer uptake assessed by standard uptake value (SUV) was generated by summation of voxels within the tomographic planes. SUV was calculated according to the formula $\left(\mathrm{C}_{\mathrm{T}} * \mathrm{~W}\right) / \mathrm{D}_{\mathrm{inj}}$, where $\mathrm{C}_{\mathrm{T}}$ is tissue radioactivity concentration, $\mathrm{W}$ is weight of the animal and $\mathrm{D}_{\mathrm{inj}}$ is injected dose. SUV max was calculated from the voxel with the highest tracer concentration.

Histologic evaluation. SCID mice used for validation were sacrificed on day 10 , the control group on day 21 and the CDDP group on day 28 after implantation by ether inhalation and cervical dislocation. Major organs (bilateral lungs, heart and mediastinal tissues) were removed, fixed in $10 \%$ formalin and embedded in paraffin. Five micrometer histologic sections were made from the lung and mediastinal tissues at $3-\mathrm{mm}$ intervals. Paraffin sections stained with hematoxylin and eosin were examined with a microscope.

\section{Experimental design}

In vivo validation of FDG PET-CT uptake. In vivo uptake of FDG in human lung cancer in the orthotopic SCID mouse model was evaluated by injecting Ma44-3 cell line into the left lungs of 6 SCID mice. Tumor volume and SUV max were calculated for all mice with FDG PET-CT ( 3 on day 9 and 3 on day 10) after implantation. All SCID mice were sacrificed at 10 days after implantation for histopathologic analysis.

Response monitoring. In vivo response monitoring was carried out by injecting Ma44-3 cell line into the right lungs of 6 SCID mice, which were divided into 2 groups; the control group $(n=3)$ and the treatment group $(n=3)$. Treatment mice were intraperitoneally injected with CDDP $(7 \mathrm{mg} / \mathrm{kg}$ body weight) on day 6 after implantation. FDG PET-CT was made on day 6, 8, 13 and 25 after implantation. Tumor volume and SUV max were calculated for all mice. The body weight of all mice was measured weekly to monitor the toxicity of CDDP. Control group mice were sacrificed at day 21, CDDP group 
Table I. Tumor volume, SUV and tumor length of 6 mice in validation of FDG uptake.

\begin{tabular}{lcccccc}
\hline Mice & 1 & 2 & 3 & 4 & 5 & 6 \\
\hline $\begin{array}{l}\text { Tumor volume } \\
\left(\mathrm{CT}, \mathrm{mm}^{3}\right)\end{array}$ & 24.40 & 9.30 & 14.60 & 33.80 & 33.90 & 25.60 \\
SUV max & 1.10 & 0.75 & 1.28 & 1.37 & 1.62 & 1.33 \\
$\begin{array}{l}\text { Tumor length } \\
(\text { specimen, mm) }\end{array}$ & 2.50 & 1.90 & 2.50 & 3.40 & 4.40 & 2.90 \\
\hline
\end{tabular}

SUV, standard uptake value; FDG, fluorodeoxyglucose, CT, computed tomography, SUV max, maximal standardized uptake value.

mice were sacrificed at day 28 after implantation for histopathologic analysis.

Statistical analysis. Comparison of tumor volume and SUV max between control group and CDDP group was calculated using unpaired Student's t-test. Correlation between SUV max, tumor volume and tumor length was calculated using linear regression. $\mathrm{P}<0.05$ was considered to indicate a statistically significant difference.

\section{Results}

In vivo validation of FDG PET-CT uptake. CT images clearly delineated tumors growing in SCID mice. Fusion of CT and PET images allowed the high uptake areas of PET scan to be matched with corresponding anatomical structure. Intense FDG uptake was demonstrated in the tumor in the left lung. Tumor volume, SUV max and tumor length of the sacrificed mice were: tumor volume, $24.3 \pm 9.3 \mathrm{~mm}^{3}$; SUV max, $1.2 \pm 0.3$ and tumor length, $2.9 \pm 0.87 \mathrm{~mm}$, as shown in Table I.

Both tumor volume and SUV max were significantly correlated with postmortem tumor length measured in specimens of 6 mice $\left(r^{2}=0.7621, P=0.023\right.$ and $r^{2}=0.8259, P=0.012$, respectively), as shown in Fig. $1 \mathrm{~A}$ and $\mathrm{B}$. Tumor volume and SUV max of 6 mice were significantly correlated $\left(r^{2}=0.6669\right.$, $\mathrm{P}=0.048$ ) as shown in Fig. 1C. Representative CT, PET-CT images are shown in Fig. 2A.

As FDG uptake of tumor in the left lung was overlapped by physiologic FDG uptake of the left ventricle, measuring a precise FDG uptake of tumor in the left lung was slightly difficult. We sought to change the implantation to the right side. The FDG uptake of tumor in the right lung was not overlapped by that of the left ventricle as shown in Fig. 2B.

Response monitoring. Representative CT, PET-CT images of the control and the CDDP group are shown in Fig. $2 \mathrm{C}$ and D. Tumor volume, SUV max and tumor length of CDDP and control mice are shown in Table II.

Tumor volume. Tumor volumes of the CDDP group were lower than the control group on day $8\left(5.43 \pm 2.08\right.$ vs. $\left.9.6 \pm 4.2 \mathrm{~mm}^{3}\right)$ although the difference was insignificant. Tumor volumes of the CDDP group were significantly lower than the control group on day $13\left(6.76 \pm 3.4\right.$ vs. $\left.53.8 \pm 24.4 \mathrm{~mm}^{3}, \mathrm{P}=0.03\right)$. Both groups
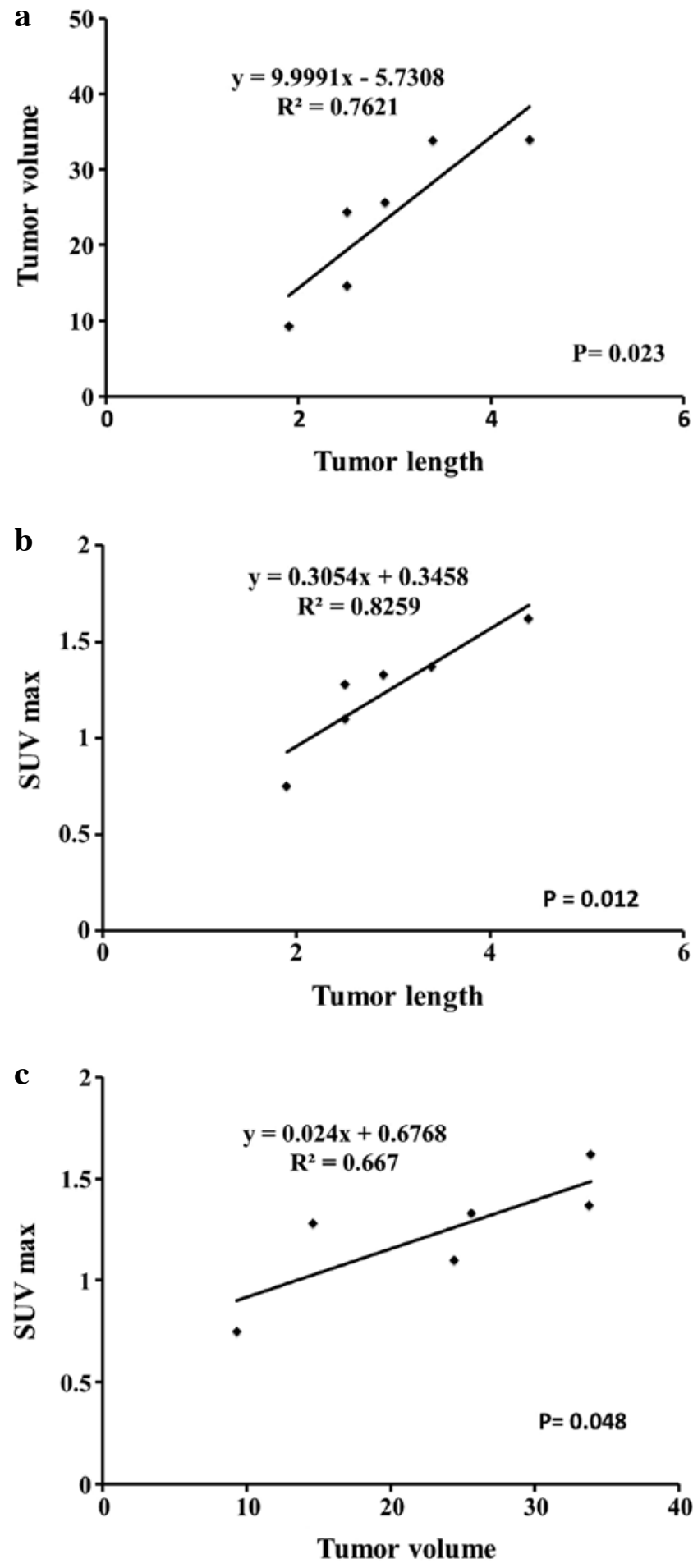

Figure 1. (a) Correlation between tumor volume $\left(\mathrm{mm}^{3}\right)$ by CT and tumor length (mm) measured in specimens. (b) Correlation between SUV max value and tumor length $(\mathrm{mm})$ measured in specimens. (c) Correlation between SUV max value and tumor volume $\left(\mathrm{mm}^{3}\right)$ measured by CT images. CT, computed tomography; SUV max, maximal standardized uptake value.

were quite similar on baseline $\left(4.53 \pm 1.8\right.$ vs. $\left.4.06 \pm 1.09 \mathrm{~mm}^{3}\right)$ as shown in Fig. 3A.

SUV max. SUV max of the CDDP group was significantly lower than that of the control group on day $8(0.469 \pm 0.08$ vs. $0.641 \pm 0.03, \mathrm{P}=0.02)$ and on day $13(0.499 \pm 0.13$ vs. $1.233 \pm 0.14$, $\mathrm{P}=0.003)$. Both groups were quite similar on baseline 
a
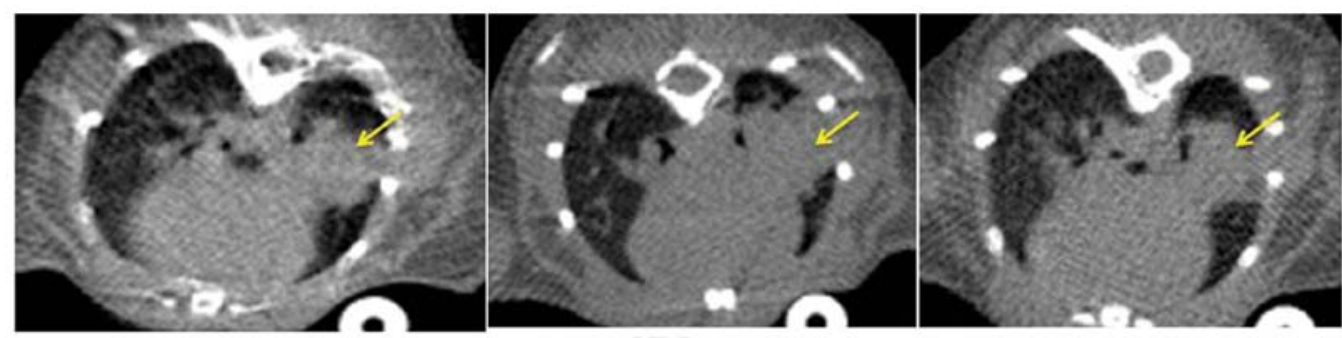

CT Images
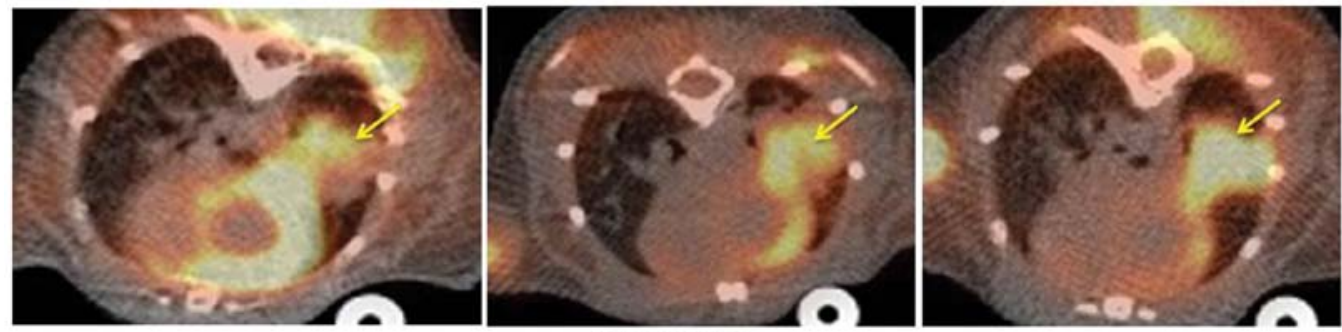

PET-CT Images

b

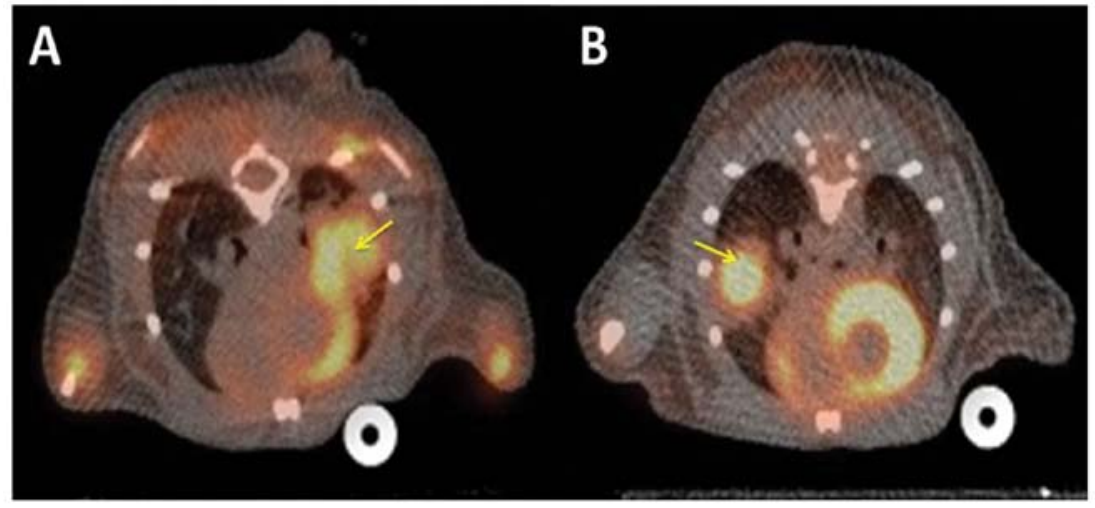

c
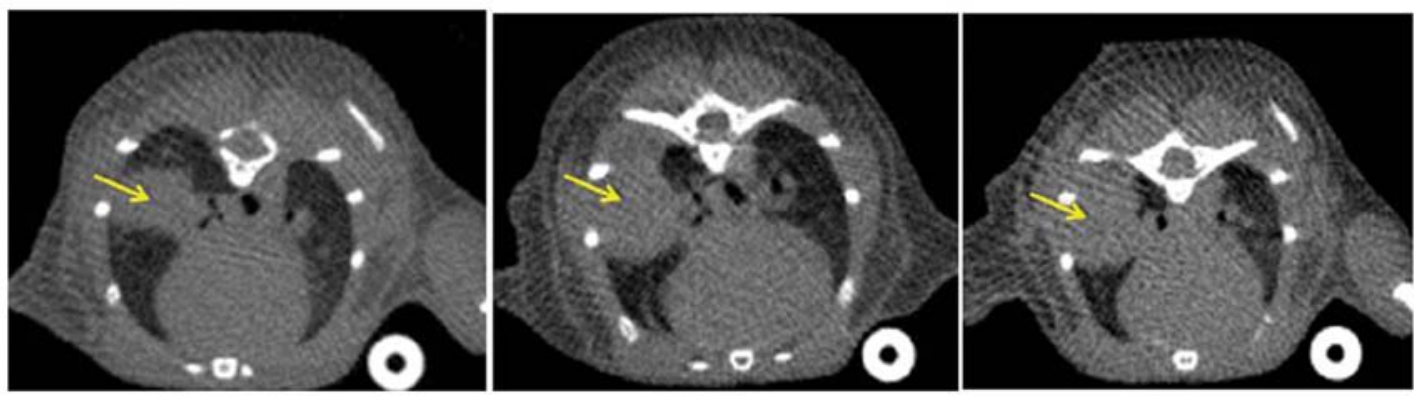

\section{Control group}
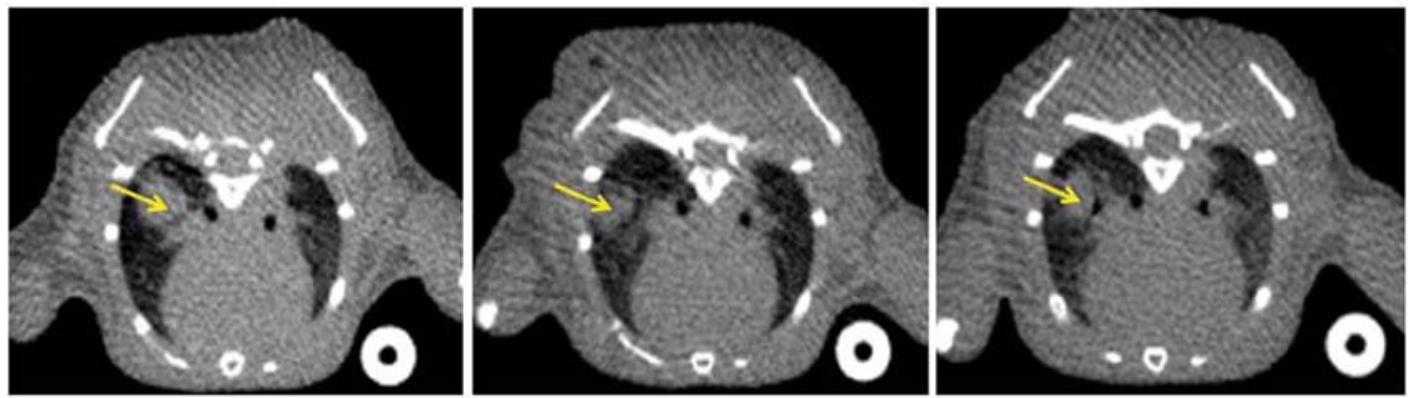

CDDP group

Figure 2. (a) An axial view of CT showing a lung tumor in the left lung (upper images), a fusion image of PET-CT showing FDG uptake in the lung tumor in the left lung (lower images); yellow arrows indicate the tumor. (b) Comparison between left and right side implantation; (A) left side, (B) right side; yellow arrows indicate the tumor. (c) An axial view of CT showing a lung tumor in the right lung of the treatment group and the control group; yellow arrows indicate the tumor. 
d
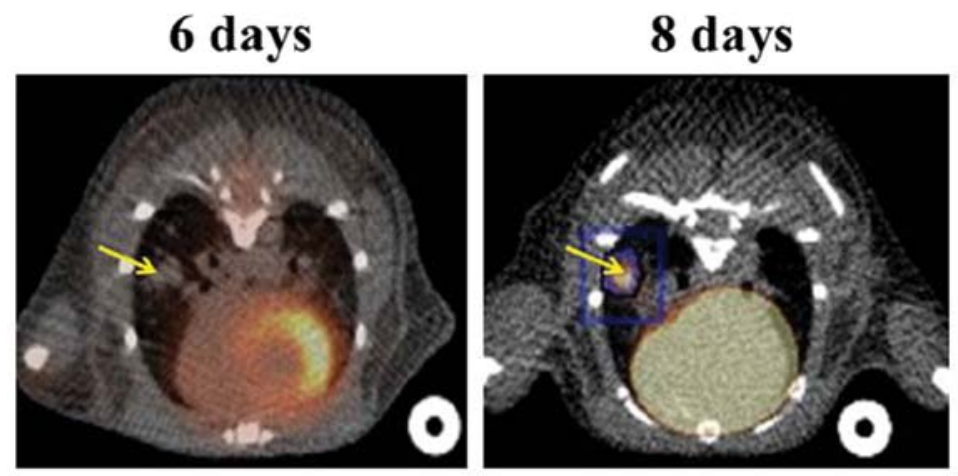

Mouse1 (control)
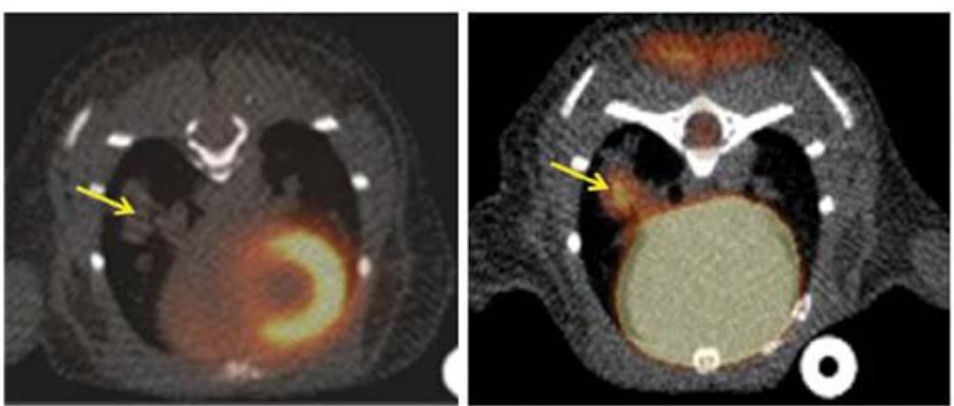

Mouse 4 (CDDP)
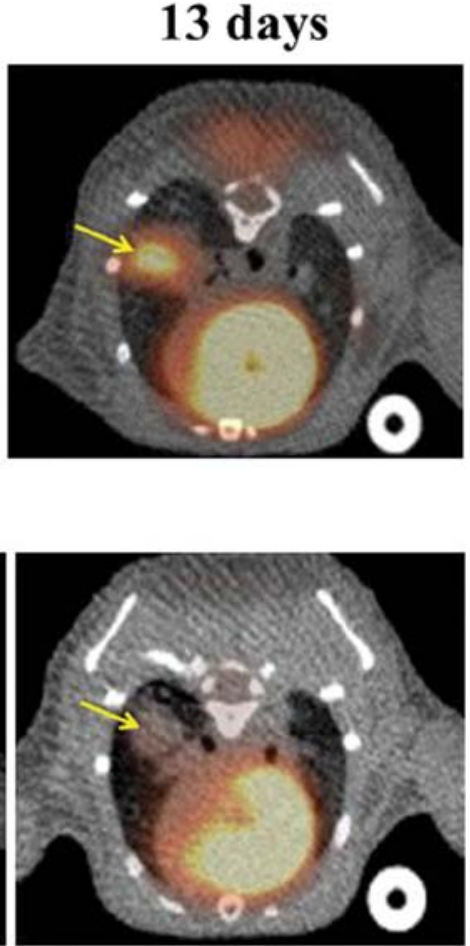

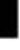

Figure 2. Continued. (d) A fusion image of PET-CT showing FDG uptake in the right lung tumor in the treatment group and the control group; yellow arrows indicate the tumor. CT, computed tomography; PET-CT, positron emission tomography-computed tomography; FDG, fluorodeoxyglucose.

Table II. Tumor volume, SUV and tumor length of implanted mice in CDDP and control group.

\begin{tabular}{lllllll}
\hline & \multicolumn{3}{c}{ CDDP group } & \multicolumn{3}{c}{ Control group } \\
\hline $\begin{array}{l}\text { Tumor } \\
\text { volume }\end{array}$ & M3 & M5 & M6 & M4 & M7 & M11 \\
Baseline & 6.5 & 2.9 & 4.2 & 3.7 & 5.3 & 3.2 \\
8 Days & 7.1 & 3.1 & 6.1 & 8.6 & 14.3 & 6.1 \\
13 Days & 10 & 3.2 & 7.1 & 25.6 & 66 & 69.8 \\
SUV max & M3 & M5 & M6 & M4 & M7 & M11 \\
Baseline & 0.552 & 0.397 & 0.43 & 0.47 & 0.49 & 0.46 \\
8 Days & 0.483 & 0.383 & 0.543 & 0.642 & 0.675 & 0.606 \\
13 Days & 0.621 & 0.355 & 0.521 & 1.102 & 1.206 & 1.391 \\
Tumor length & 8.8 & 7.6 & 6.5 & 7.7 & Friable & 11.2 \\
& & & & & tumor & \\
\hline
\end{tabular}

SUV, standard uptake value; CDDP, cisplatin; SUV max, maximal standardized uptake value.

$(0.456 \pm 0.08$ vs. $0.474 \pm 0.015)$ as shown in Fig. 3B. SUV max of the CDDP group was elevated on day 25 reaching $1.696 \pm 0.12$ (data not shown).

Correlation between SUV max and tumor volume measured by CT images. SUV max of the CDDP group was significantly correlated with tumor volume measured by CT $\left(\mathrm{r}^{2}=0.9784\right.$, $\mathrm{P}=0.0002$ ) as shown in Fig. 3C. SUV max of the control group was significantly correlated with tumor volume measured by CT $\left(r^{2}=0.8671, P=0.0069\right)$ as shown in Fig. 3D.

Correlation between SUV max and tumor length measured in specimens. SUV max of the CDDP group was significantly correlated with tumor length measured in specimens $(\mathrm{P}=0.0093)$. In the control group, one mouse died before being sacrificed and tumor tissue was too friable to prepare slide. The number of mice became too few to test for significant correlation.

Body weight. There were no marked changes in body weight between the CDDP group and the control group until day 8 after implantation. The body weight of the mice in the CDDP group on day 13 was significantly lower than that in the control group (21.133 vs. $24.067 \mathrm{~g}, \mathrm{P}=0.017)$. However, the body weight of the mice in the CDDP group on day 19 was significantly higher than that in the control group (24.00 vs. $21.350 \mathrm{~g}, \mathrm{P}=0.018$ ) as shown in Fig. 3E.

Histopathology. Tumors were histopathologically confirmed in the lungs of all mice. In the CDDP group specimens, we observed some regions where most of the tumor cells showed nuclear pycnosis consistent with necrosis. However, there was no such region in the control group (Fig. 4A and B). The tumor length measured in specimens for the CDDP group (sacrificed on day 28) was $1.696 \pm 0.15$, the tumor length measured in specimens for the control group (sacrificed on day 21) was $1.24 \pm 0.14$, one mouse of the control group died before being sacrificed and the tumor was too friable to be prepared for histopathological slides. 


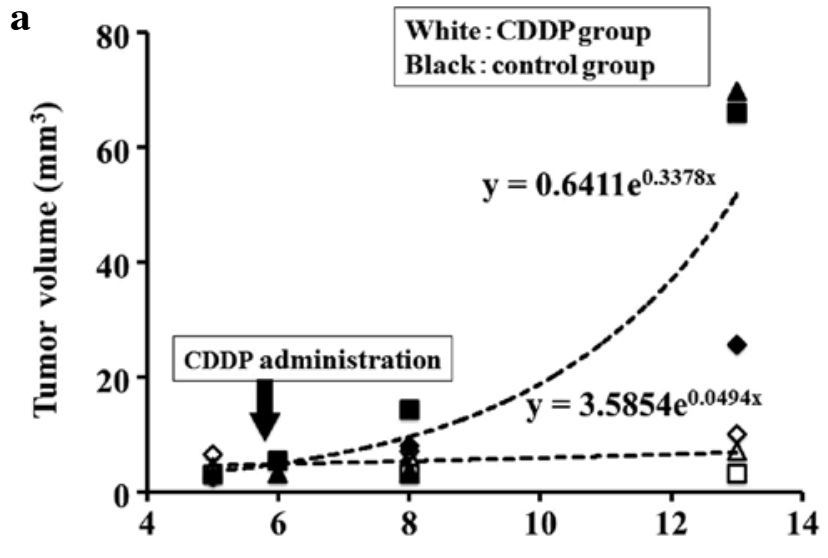

Time after implantation

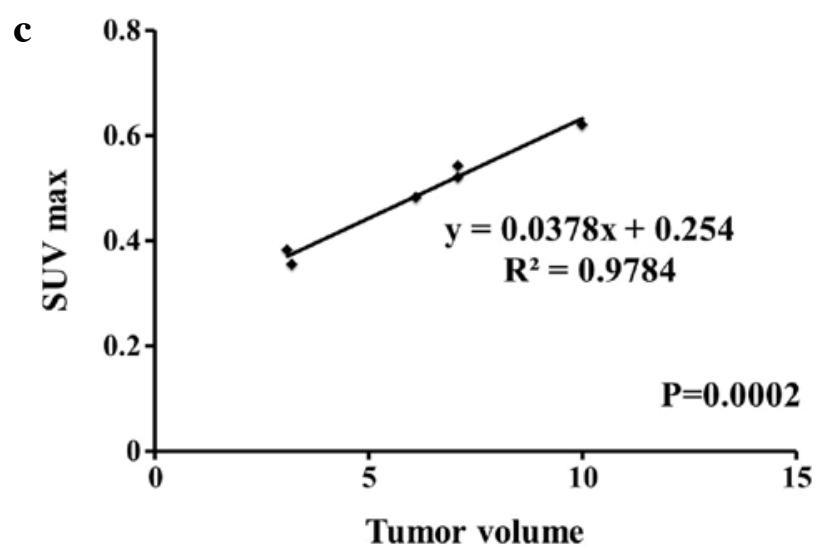

e

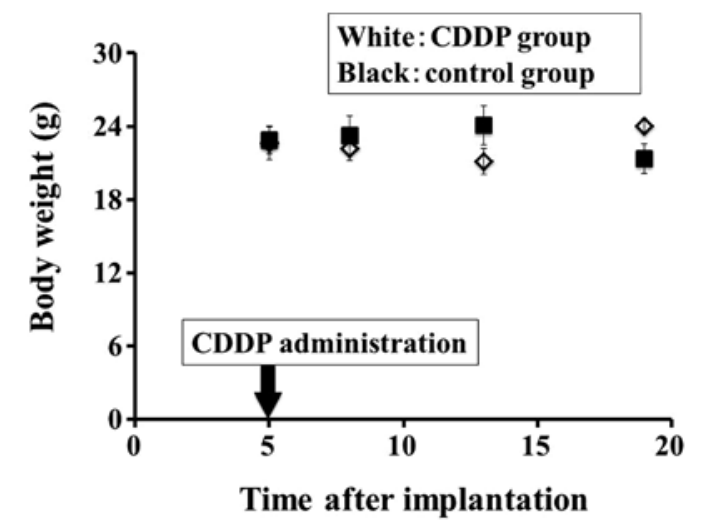

\section{Discussion}

In 1889, Paget postulated that an organ-specific site presumably provides tumor cells with the most appropriate environment for local growth and metastasis 'seed and soil' theory (22). Considerable effort has been made to develop more clinically relevant models by the use of orthotopic implantation models (5). We have established an orthotopic lung cancer model (1,6-11). Our model was simple, easy and reproducible. Many models can be produced at once. The main downside of this model is that tumor size or tumor volume changes are more difficult to continuously monitor reproducibility, except at necropsy $(5,12)$. Thus, to overcome this shortcoming we used small animal imaging, which is a non-invasive and repeatable method for monitoring the course of disease under therapy.

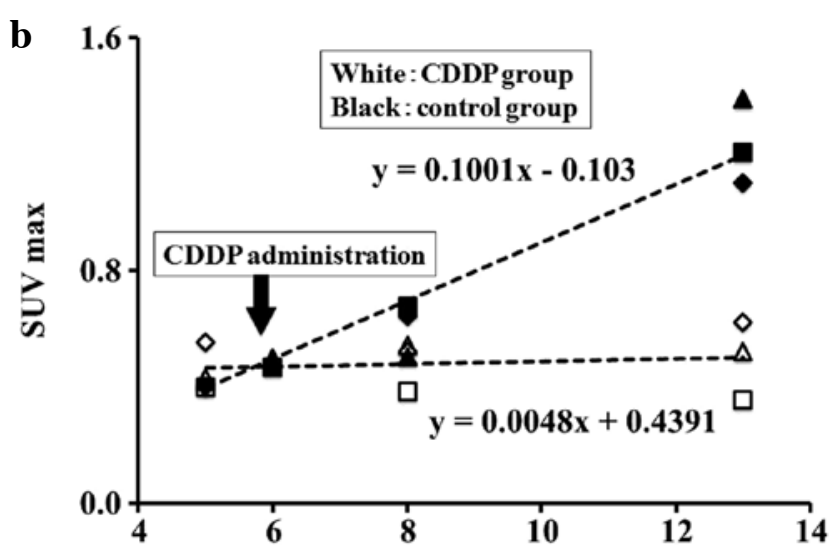

Time after implantation

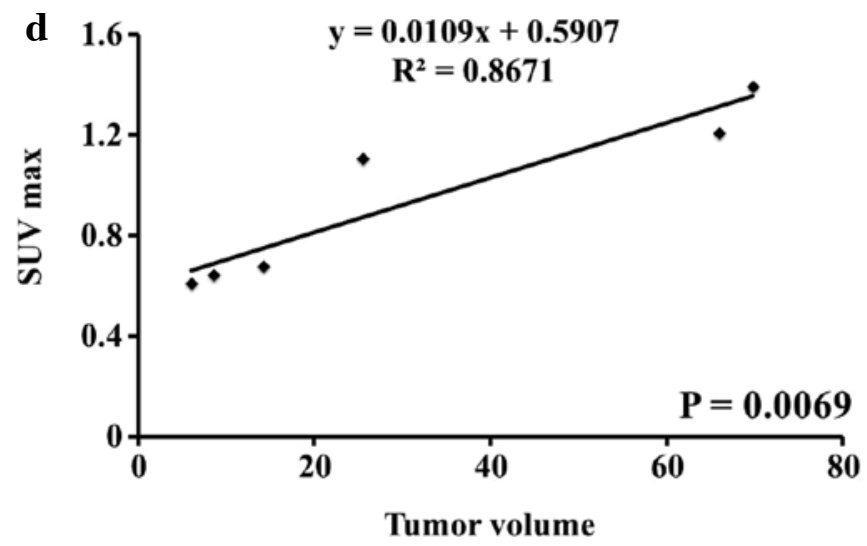

Figure 3. (a) Tumor volume change in relation to time after implantation (white, treatment group; black, control group). (b) SUV max in relation to time after implantation (white, treatment group; black, control group). (c) Correlation between SUV max value and tumor volume $\left(\mathrm{mm}^{3}\right)$ measured by CT images in the CDDP group. (d) Correlation between SUV max value and tumor volume $\left(\mathrm{mm}^{3}\right)$ measured by CT images in the control group. (e) Body weight of mice in relation to time after implantation (white, treatment group; black, control group). SUV max, maximal standardized uptake value; $\mathrm{CT}$, computed tomography; CDDP, cisplatin.

The present study demonstrated the feasibility of using FDG PET-CT to evaluate repeatedly and non-invasively tumor progression in an orthotopic lung cancer model. Validation of in vivo FDG uptake in the orthotopic lung cancer model revealed that FDG uptake predicted tumor volume and size. Both tumor volume and SUV max were significantly correlated with postmortem tumor length measured in specimens $(\mathrm{P}=0.023, \mathrm{P}=0.012)$. Tumor volume and SUV $\max$ were significantly correlated $(\mathrm{P}=0.048)$. SUV max was more sensitive than tumor volume in prediction of tumor size. Therefore, the present study confirmed that FDG PET-CT is a valuable and reliable tool in staging progression of lung cancer in an orthotopic model without needing to sacrifice more mice. Many studies reported that combined PET-CT allows the acquisition of functional PET images and morphological CT 
$\mathbf{a}$
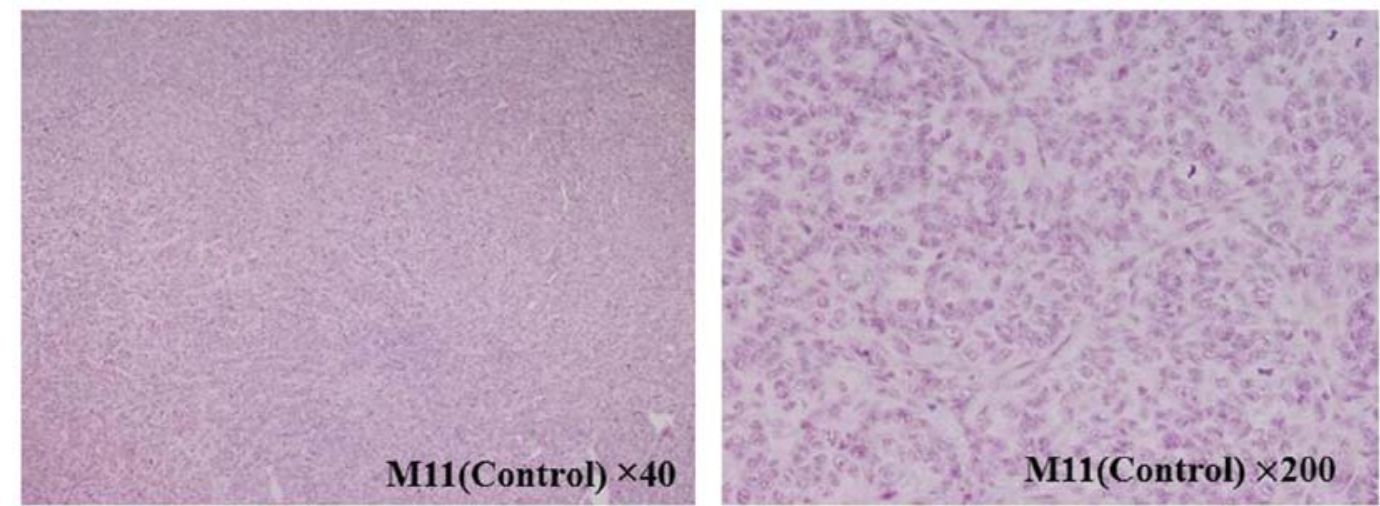

b
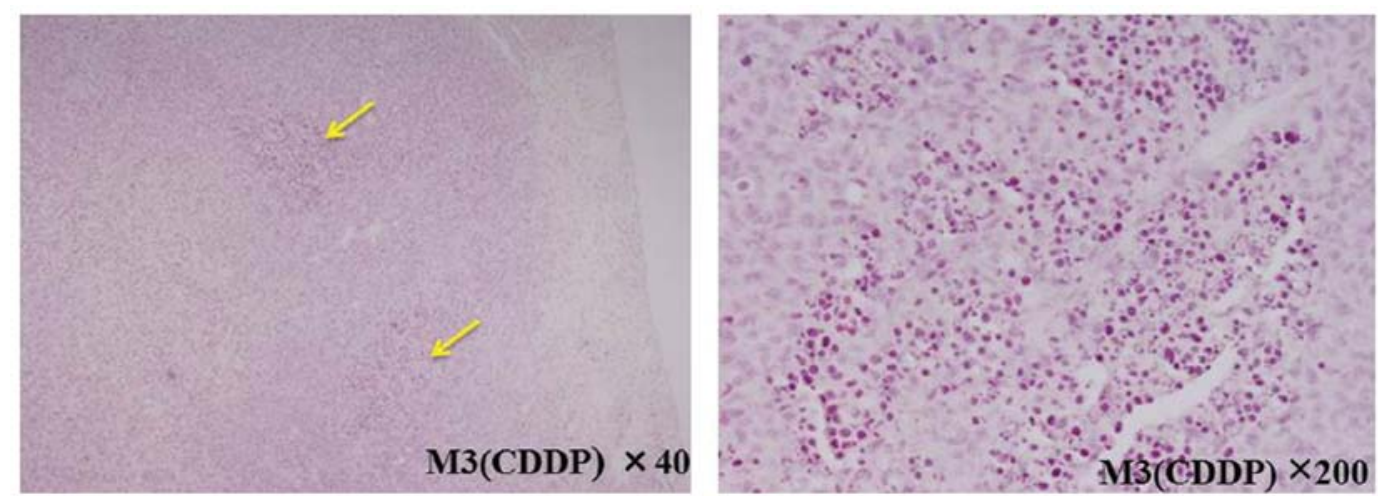

Figure 4. (a) Histopathology of control group representative case magnified $x 40$ and x200; most of the tumor cells showed no pycnosis. (b) Histopathology of CDDP group representative case magnified $\mathrm{x} 40$ and $\mathrm{x} 200$; most of the tumor cells showed nuclear pycnosis consistent with necrosis. CDDP, cisplatin.

images, thus the areas of tracer uptake can be better localized to the corresponding anatomical structure, resulting in improved sensitivity and specificity in tumor imaging (23-25).

The present study demonstrated the reproducibility of FDG PET-CT in monitoring repeatedly and non-invasively the inhibitory effect of CDDP on tumor growth in an orthotopic model without the need to sacrifice more mice. SUV max of the CDDP group was significantly lower than the control group on day $8(\mathrm{P}=0.02)$ and on day $13(\mathrm{P}=0.003)$. Tumor volume of the CDDP group was lower than the control group on day 8 and significantly lower on day $13(\mathrm{P}=0.03)$. Changes in FDG signal appeared earlier than changes in tumor volume by CT; significant difference in SUV max between CDDP group and control group on day $8(\mathrm{P}=0.02)$ appeared earlier than tumor volume that showed no significant difference on day 8 , and that was consistent with the general concept that metabolic changes appear earlier than morphological changes $(26,27)$. Thus, the present study may overcome the drawbacks of tumor volume changes which occur late after therapy impeding quick decision in case of non-response. In the treatment group, there was no regression in tumor volume, but static or minor elevation very close to baseline. As we used CDDP as a single agent it will be very difficult to cause tumor shrinkage. Therefore, it may exert tumor stasis rather than tumor shrinkage. Several novel anticancer drugs are cytostatic and do not necessarily lead to reduction of tumor volume, but of viable tumor tissue (28). Identification of the effect with drugs exerting tumor stasis can be difficult, as the conventional anatomical imaging modalities CT and MRI measure treatment effect by assessing changes in tumor size.
A tumor stasis effect of anticancer treatment can consequently be missed by these anatomical imaging modules. Therefore, identification of biological biomarkers is of great value in treatment regimens involving tumoristatic compounds (29). In the present study, FDG uptake changes following treatment predicted changes in tumor volume and size supporting the potential use of SUV max as a predictive parameter of tumor response to chemotherapy and a sensitive marker for tumor staging in preclinical trials.

SUV max of the CDDP group increased to $1.69 \pm 0.12$ on day 25; we can explain the increase in SUV max in our results by our use of CDDP as monotherapy and it may be less effective and does not inhibit glucose uptake in the most aggressive parts of the tumor. CDDP monotherapy can maintain reduction in FDG uptake until day 13 after implantation but cannot maintain reduction until day 25 . This may be due to the proliferation of CDDP-resistant cells after most sensitive cells were killed during the early therapeutic response.

The right side implantation was better in the discrimination of tumor uptake from the heart uptake than the left side. SUV max of the right side was significantly correlated with tumor volume $(\mathrm{P}=0.0002, \mathrm{P}=0.0069)$ compared with that in the left side $(\mathrm{P}=0.048)$.

In our model, we detected CDDP toxicity by measuring mouse body weight; the body weight of the CDDP group on day 13 was significantly lower than that of the control group due to CDDP toxicity $(\mathrm{P}=0.017)$. However, the body weight of the CDDP group on day 19 was significantly higher than that of the control group $(\mathrm{P}=0.018)$ due to cachexia associated with advanced tumor. 
In conclusion, the present study provided additional support for using FDG PET-CT in the detection of tumor progression and therapeutic response of lung cancer in an orthotopic model non-invasively and repeatedly. Furthermore, our design overcame the drawbacks of orthotopic models and traditional anatomical imaging modalities.

\section{Acknowledgements}

This study was supported by the Grants-in-Aid for Scientific Research from the Ministry of Education, Culture, Sports, Science and Technology (24659634).

\section{References}

1. Fujino $\mathrm{H}$, Kondo $\mathrm{K}$, Ishikura $\mathrm{H}$, et al: Matrix metalloproteinase inhibitor MMI-166 inhibits lymphogenous metastasis in an orthotopically implanted model of lung cancer. Mol Cancer Ther 4: 1409-1416, 2005.

2. Oliver TG, Mercer KL, Sayles LC, et al: Chronic cisplatin treatment promotes enhanced damage repair and tumor progression in a mouse model of lung cancer. Genes Dev 24: 837-852, 2010.

3. Kelland L: The resurgence of platinum-based cancer chemotherapy. Nat Rev Cancer 7: 573-584, 2007.

4. Francia G, Cruz-Munoz W, Man S, et al: Mouse models of advanced spontaneous metastasis for experimental therapeutics. Nat Rev Cancer 11: 135-141, 2011.

5. Bibby MC: Orthotopic models of cancer for preclinical drug evaluation: advantages and disadvantages. Eur J Cancer 40 852-857, 2004.

6. Miyoshi T, Kondo K, Ishikura H, et al: SCID mouse lymphogenous metastatic model of human lung cancer constructed using orthotopic inoculation of cancer cells. Anticancer Res 20: 161-163, 2000.

7. Ishikura H, Kondo K, Miyoshi T, et al: Artificial lymphogenous metastatic model using orthotopic implantation of human lung cancer. Ann Thorac Surg 69: 1691-1695, 2000.

8. Ishikura H,Kondo K, Miyoshi T, et al: Suppression of mediastinal metastasis by uracil-tegafur or $c i s$-diamminedichloroplatinum(II) using a lymphogenous metastatic model in a human lung cancer cell line. Clin Cancer Res 7: 4202-4208, 2001.

9. Fujino H, Kondo K, Miyoshi T, et al: Establishment of patientlike SCID mouse model by orthotopically implanting primary cultured cells from surgically-resected lung cancer tissues. Oncol Rep 10: 1709-1715, 2003.

10. Ishikura H, Kondo K, Miyoshi T, et al: Green fluorescent protein expression and visualization of mediastinal lymph node metastasis of human lung cancer cell line using orthotopic implantation. Anticancer Res 24: 719-723, 2004

11. Kondo K, Fujino H, Miyoshi T, et al: Orthotopically implanted SCID mouse model of human lung cancer suitable for investigating metastatic potential and anticancer drug effects. Oncol Rep 12: 991-999, 2004.
12. Zhang $X$ and $\mathrm{Wu} \mathrm{J}$ : Establishing of the transplanted animal models for human lung cancer. JNMU 23: 1-5, 2009.

13. Myers R: The biological application of small animal PET imaging. Nucl Med Biol 28: 585-593, 2001.

14. Chatziioannou AF: Molecular imaging of small animals with dedicated PET tomographs. Eur J Nucl Med Mol Imaging 29: 98-114, 2002.

15. Wahl RL, Jacene H, Kasamon Y, et al: From RECIST to PERCIST: evolving considerations for PET response criteria in solid tumors. J Nucl Med 50 (Suppl 1): 122S-150S, 2009.

16. Pauwels EK, Sturm EJ, Bombardieri E, et al: Positron-emission tomography with $\left[{ }^{18} \mathrm{~F}\right]$ fluorodeoxyglucose. Part I. Biochemical uptake mechanism and its implication for clinical studies. J Cancer Res Clin Oncol 126: 549-559, 2000.

17. Kostakoglu L, Hardoff R, Mirtcheva R and Goldsmith SJ: PET-CT fusion imaging in differentiating physiologic from pathologic FDG uptake. Radiographics 24: 1411-1431, 2004.

18. Dose Schwarz J, Bader M, Jenicke L, et al: Early prediction of response to chemotherapy in metastatic breast cancer using sequential ${ }^{18} \mathrm{~F}-\mathrm{FDG}$ PET. J Nucl Med 46: 1144-1150, 2005.

19. von Falck C, Maecker B, Schirg E, et al: Post transplant lymphoproliferative disease in pediatric solid organ transplant patients: a possible role for $\left[{ }^{18} \mathrm{~F}\right]-\mathrm{FDG}-\mathrm{PET}(/ \mathrm{CT})$ in initial staging and therapy monitoring. Eur J Radiol 63: 427-435, 2007.

20. Fueger BJ, Czernin J, Hildebrandt I, et al: Impact of animal handling on the results of ${ }^{18} \mathrm{~F}$-FDG PET studies in mice. J Nucl Med 47: 999-1006, 2006.

21. Jensen MM, Erichsen KD, Björkling F, et al: Early detection of response to experimental chemotherapeutic Top216 with $\left[{ }^{18} \mathrm{~F}\right]$ FLT and $\left[{ }^{18} \mathrm{~F}\right] \mathrm{FDG}$ PET in human ovary cancer xenografts in mice. PLoS One 5: e12965, 2010.

22. Paget S: The distribution of secondary growths in cancer of the breast. Lancet 133: 571-573, 1889.

23. Beyer T, Townsend DW and Blodgett TM: Dual-modality PET/CT tomography for clinical oncology. Q J Nucl Med 46: 24-34, 2002.

24. Ell PJ and von Schulthess GK: PET/CT: a new road map. Eur J Nucl Med Mol Imaging 29: 719-720, 2002.

25. Tatsumi M, Nakamoto Y, Traughber B, et al: Initial experience in small animal tumor imaging with a clinical positron emission tomography/computed tomography scanner using 2-[F-18]fluoro2-deoxy-D-glucose. Cancer Res 63: 6252-6257, 2003.

26. Kawada K, Murakami K, Sato T, et al: Prospective study of positron emission tomography for evaluation of the activity of lapatinib, a dual inhibitor of the ErbB1 and ErbB2 tyrosine kinases, in patients with advanced tumors. Jpn J Clin Oncol 37: 44-48, 2007.

27. Kostakoglu L and Goldsmith SJ: ${ }^{18}$ F-FDG PET evaluation of the response to therapy for lymphoma and for breast, lung, and colorectal carcinoma. J Nucl Med 44: 224-239, 2003.

28. Barthel H, Cleij MC, Collingridge DR, et al: 3'-Deoxy- 3 '- $\left[{ }^{18} \mathrm{~F}\right]$ fluorothymidine as a new marker for monitoring tumor response to antiproliferative therapy in vivo with positron emission tomography. Cancer Res 63: 3791-3798, 2003.

29. Stimson L and La Thangue NB: Biomarkers for predicting clinical responses to HDAC inhibitors. Cancer Lett 280: 177-183, 2009. 